\title{
Fullerene-like Chemistry at the Interior Carbon Atoms of an Alkene-centered $\mathrm{C}_{26} \mathrm{H}_{12}$ Geodesic Polyarene
}

\author{
Hindy E. Bronstein ${ }^{* \dagger}$ and Lawrence T. Scott* $*$ \\ Department of Natural Science, Fordham University, 113 West 60th St., LL 817-F, New York, NY \\ 10023 and Department of Chemistry, Merkert Chemistry Center, Boston College, Chestnut Hill, MA \\ 02467-3860
}

\section{Supporting Information}

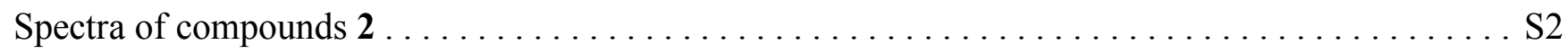

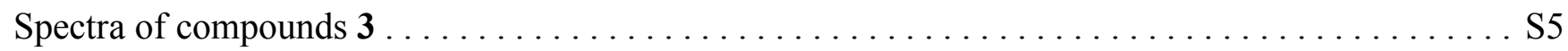

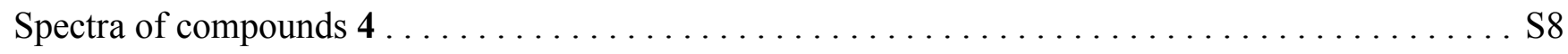

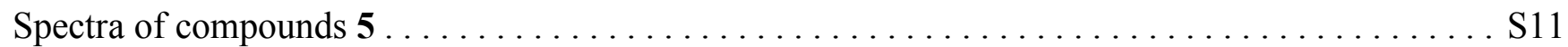

Calculated DFT (B3LYP/6-31G*) energies and X,Y,Z-coordinates for

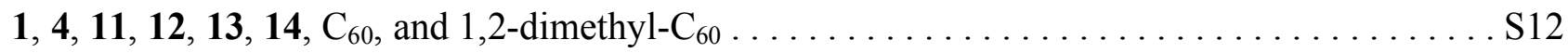




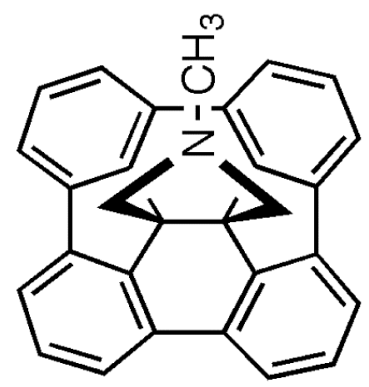

N

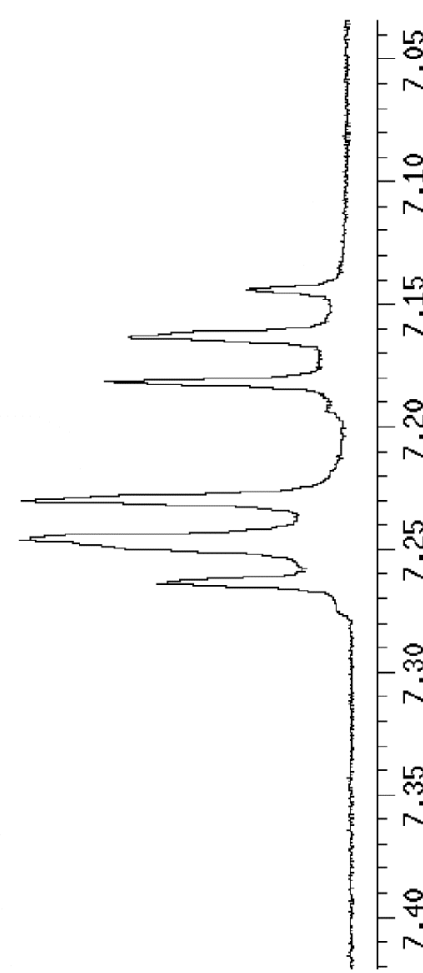

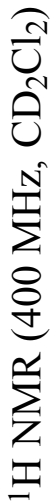

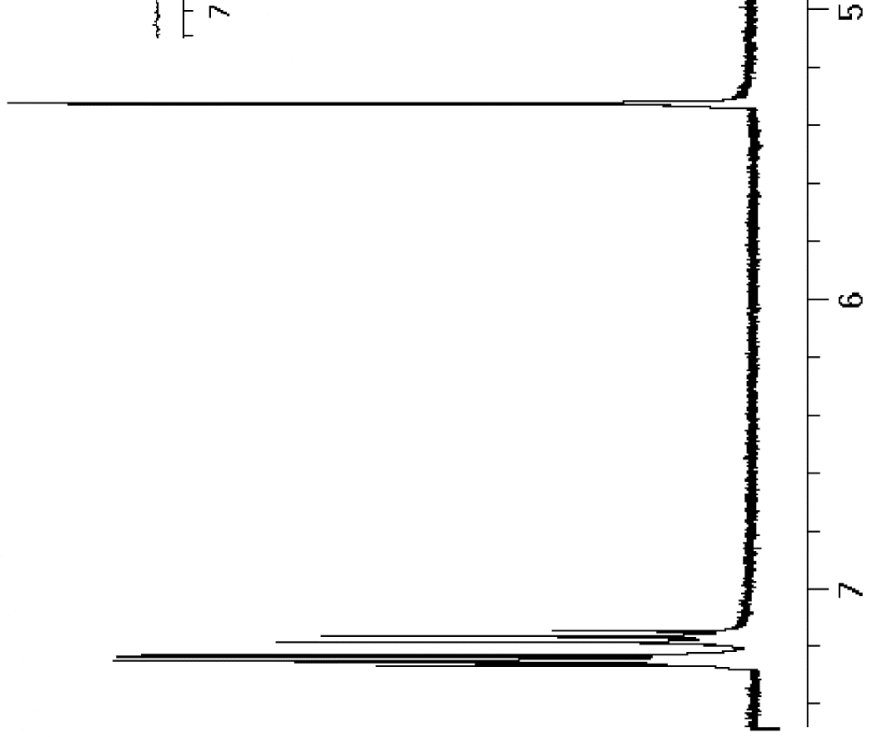




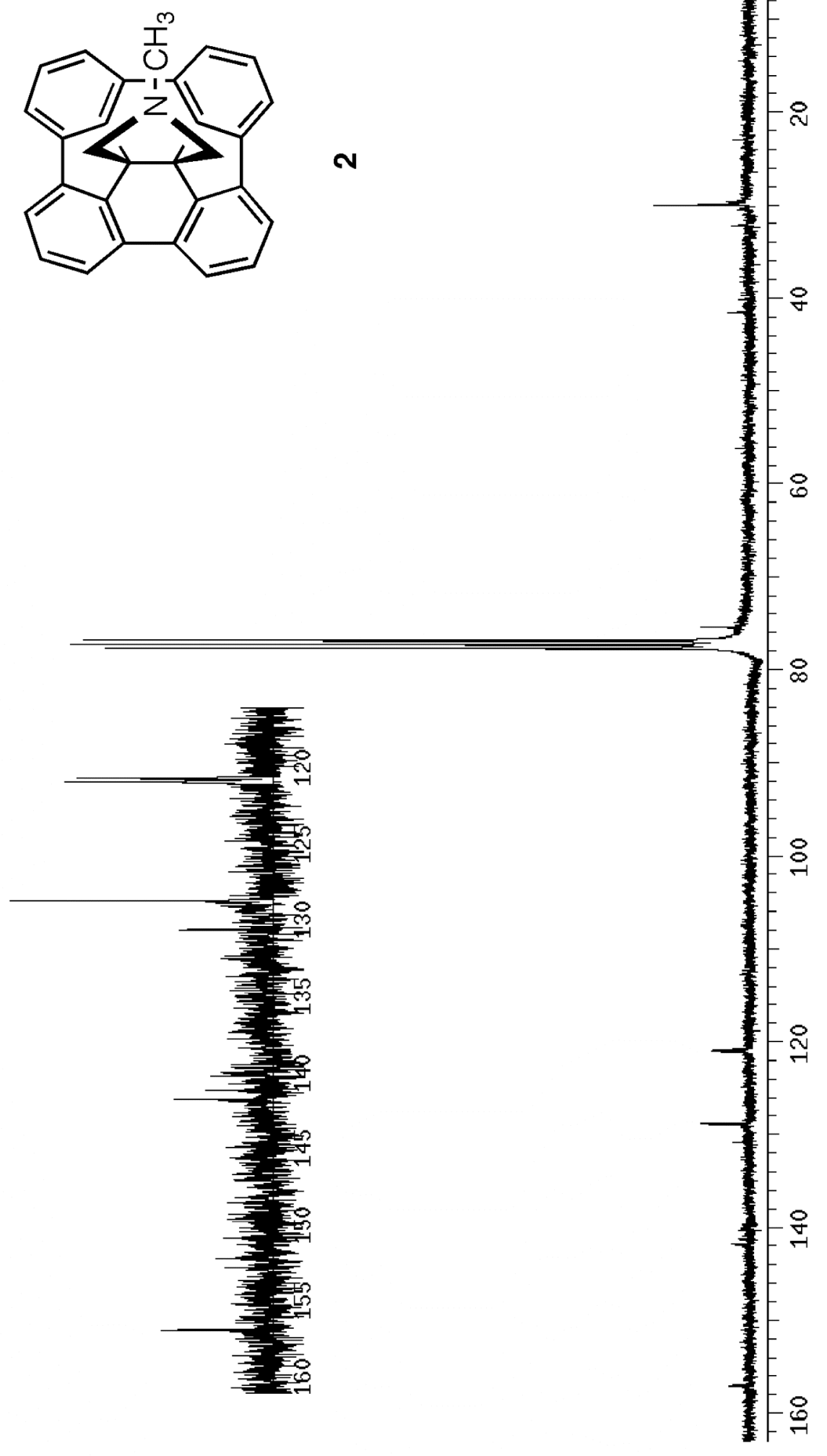

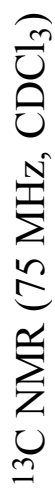




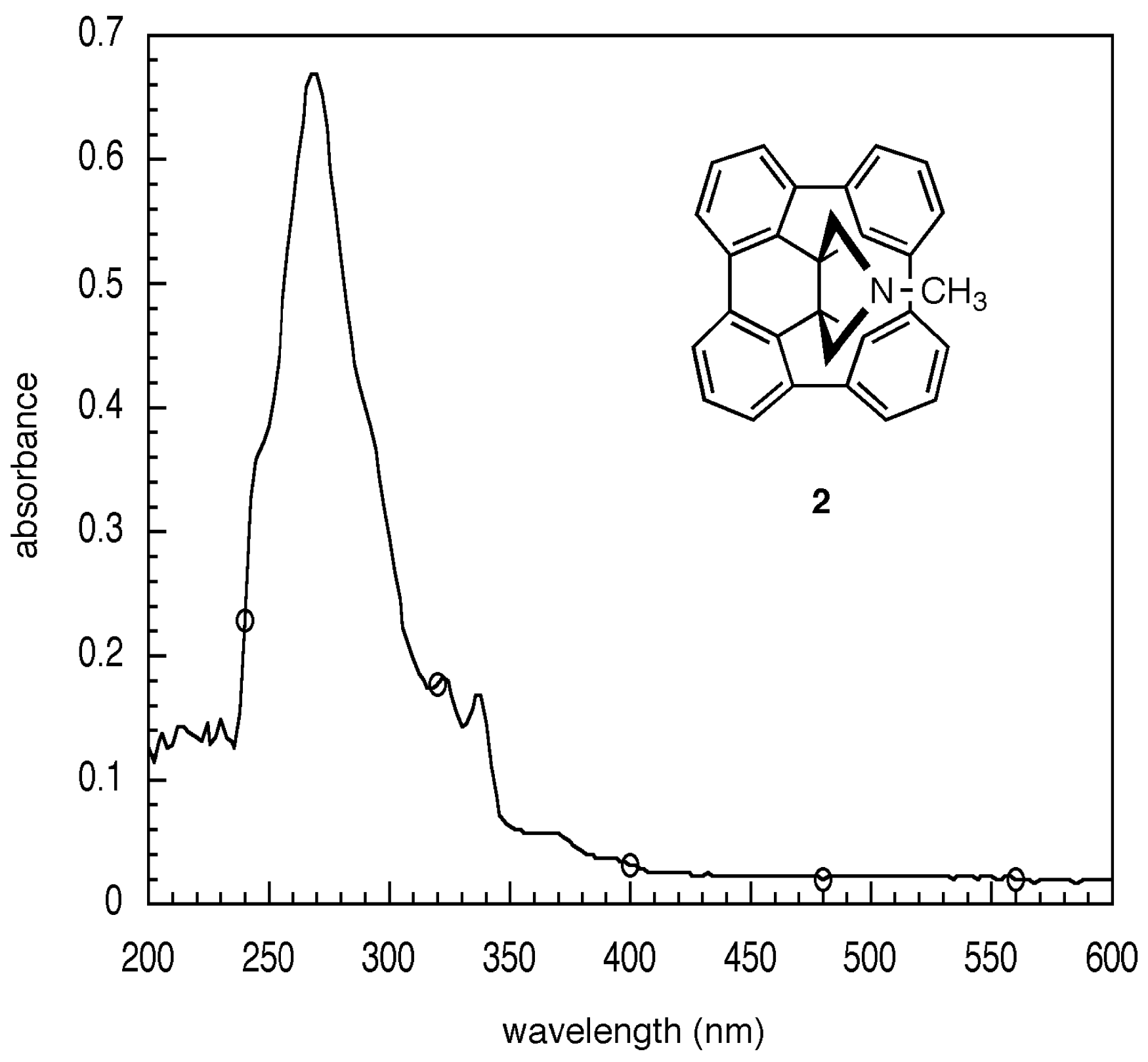

UV-vis $\left(\mathrm{CHCl}_{3}\right)$ 


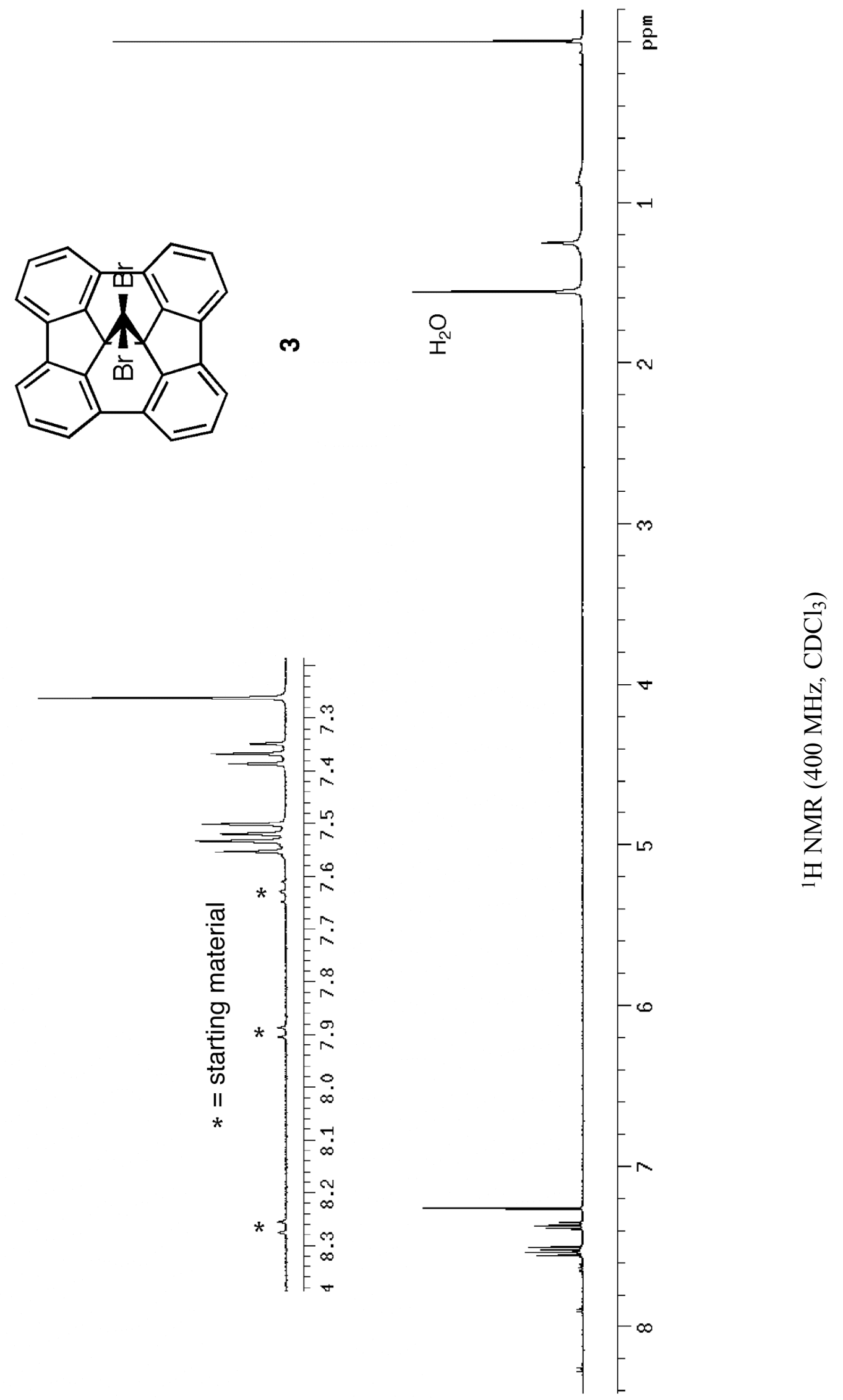




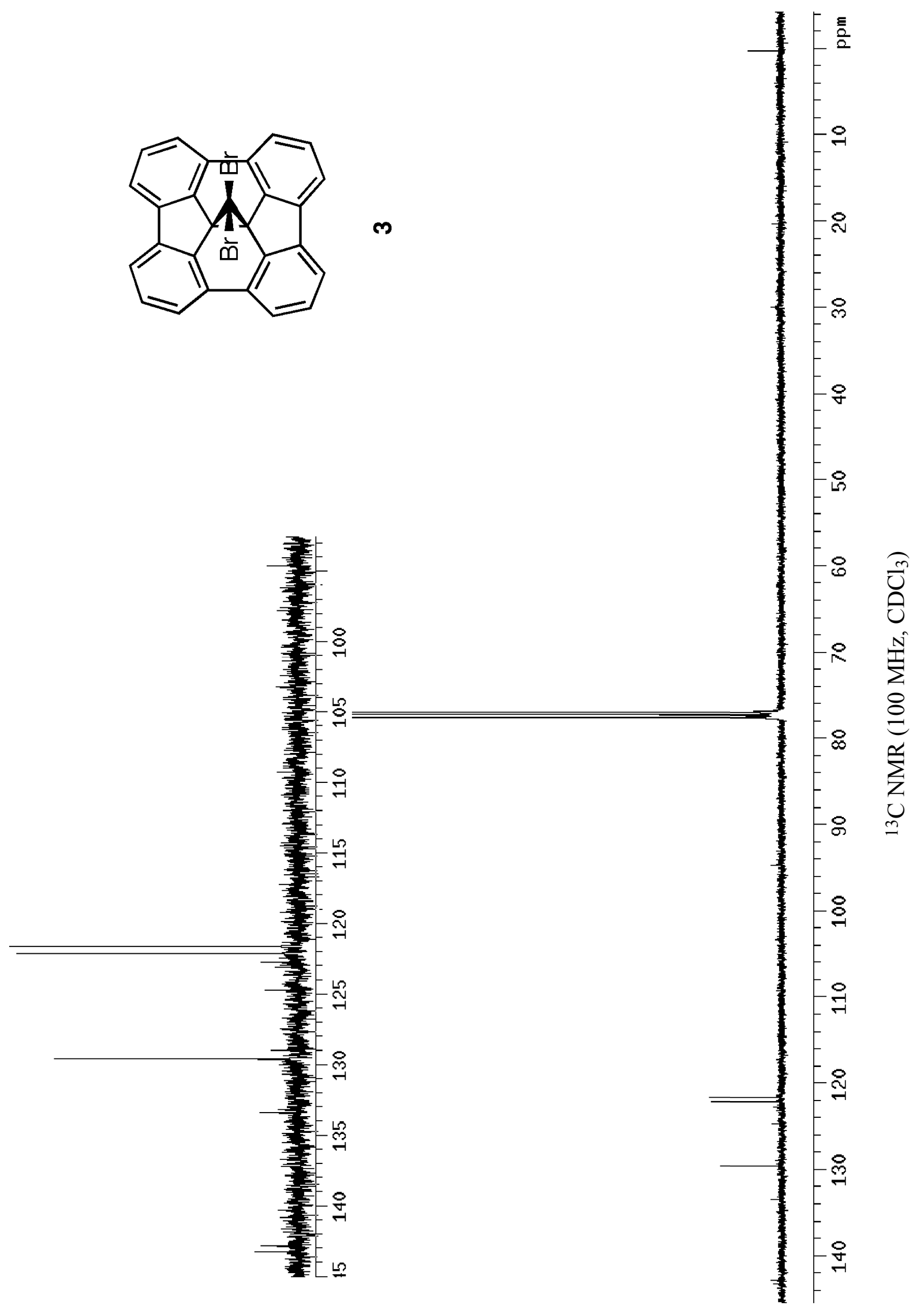




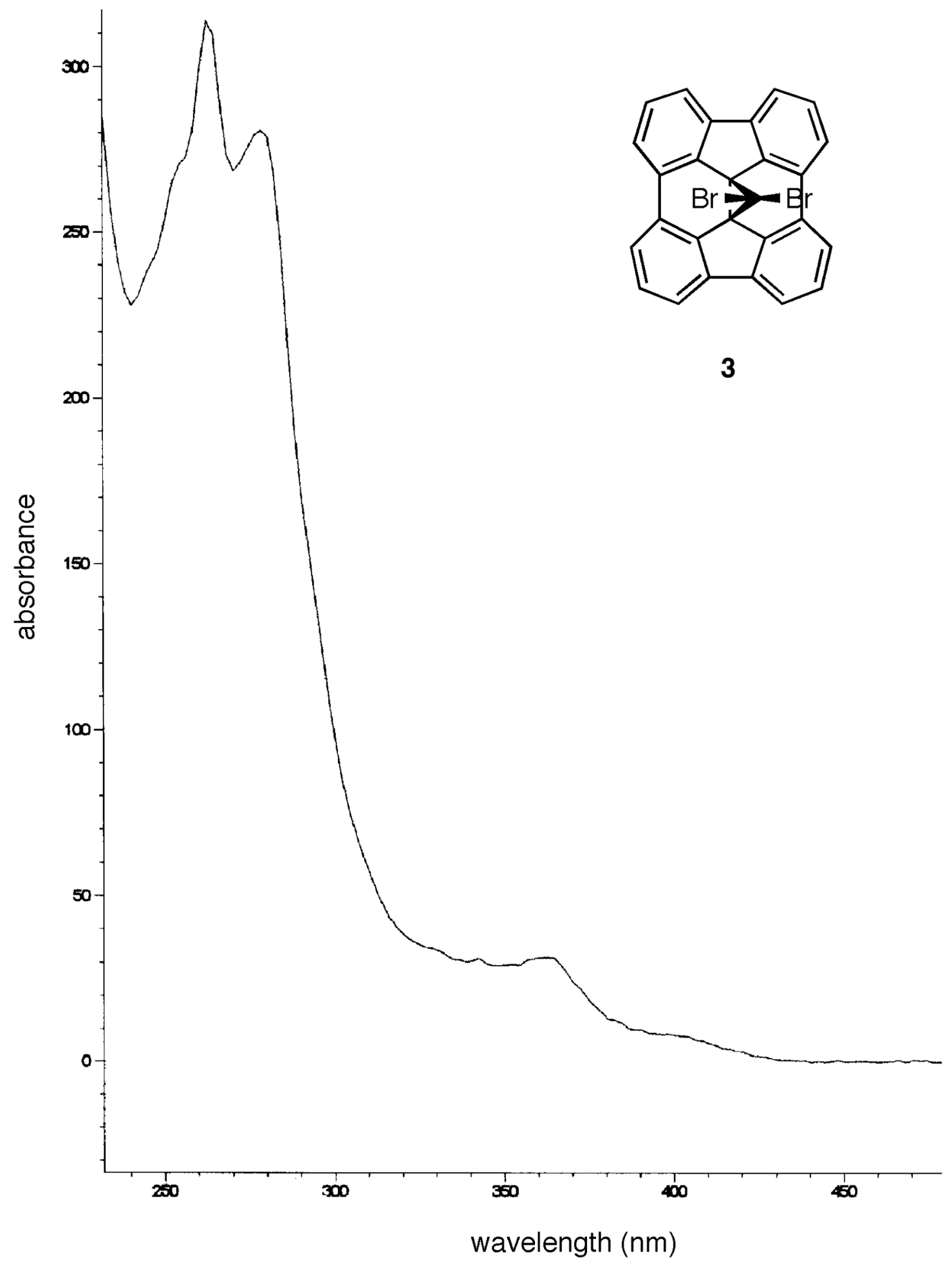

UV-vis $\left(\mathrm{CH}_{2} \mathrm{Cl}_{2}\right)$ 


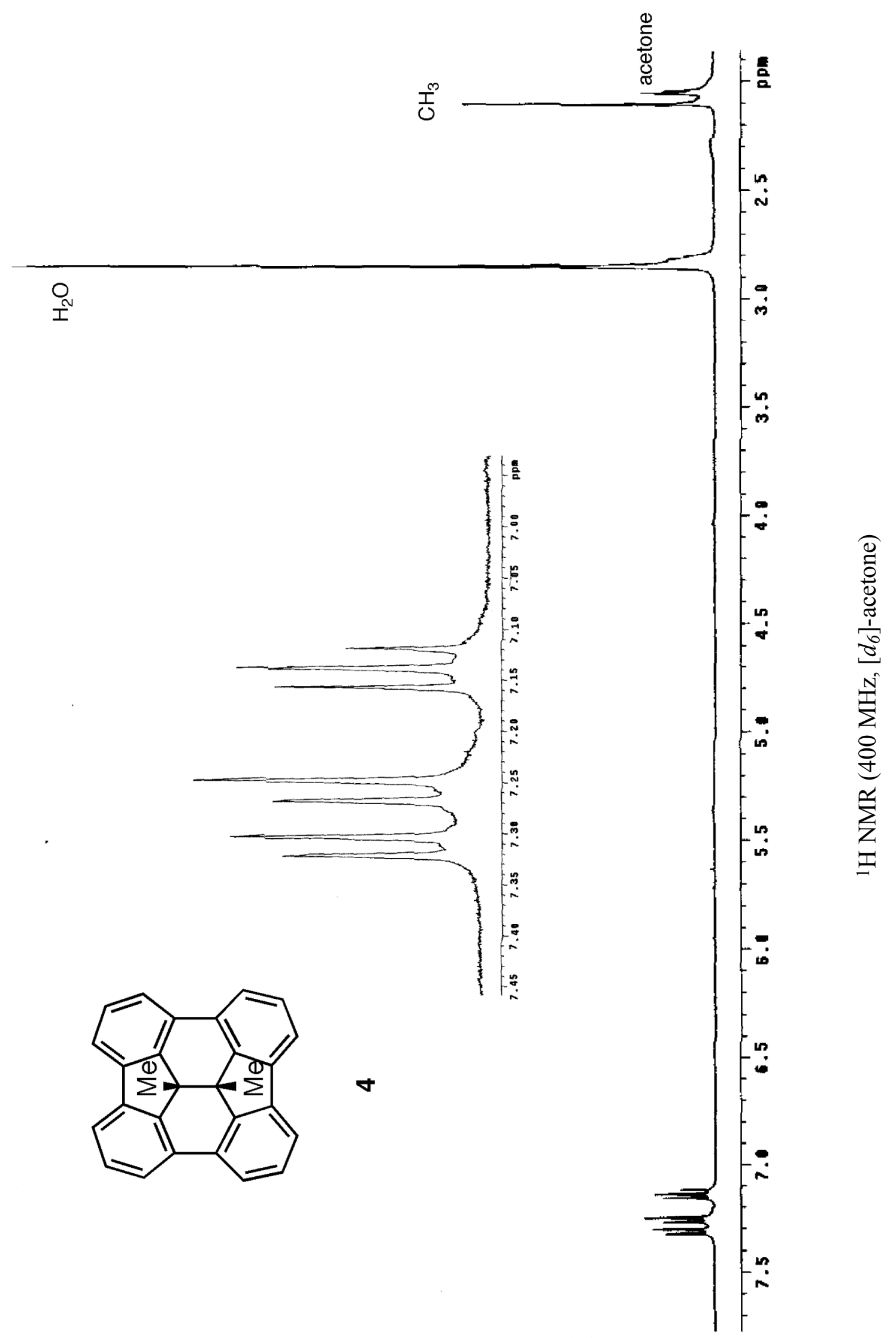




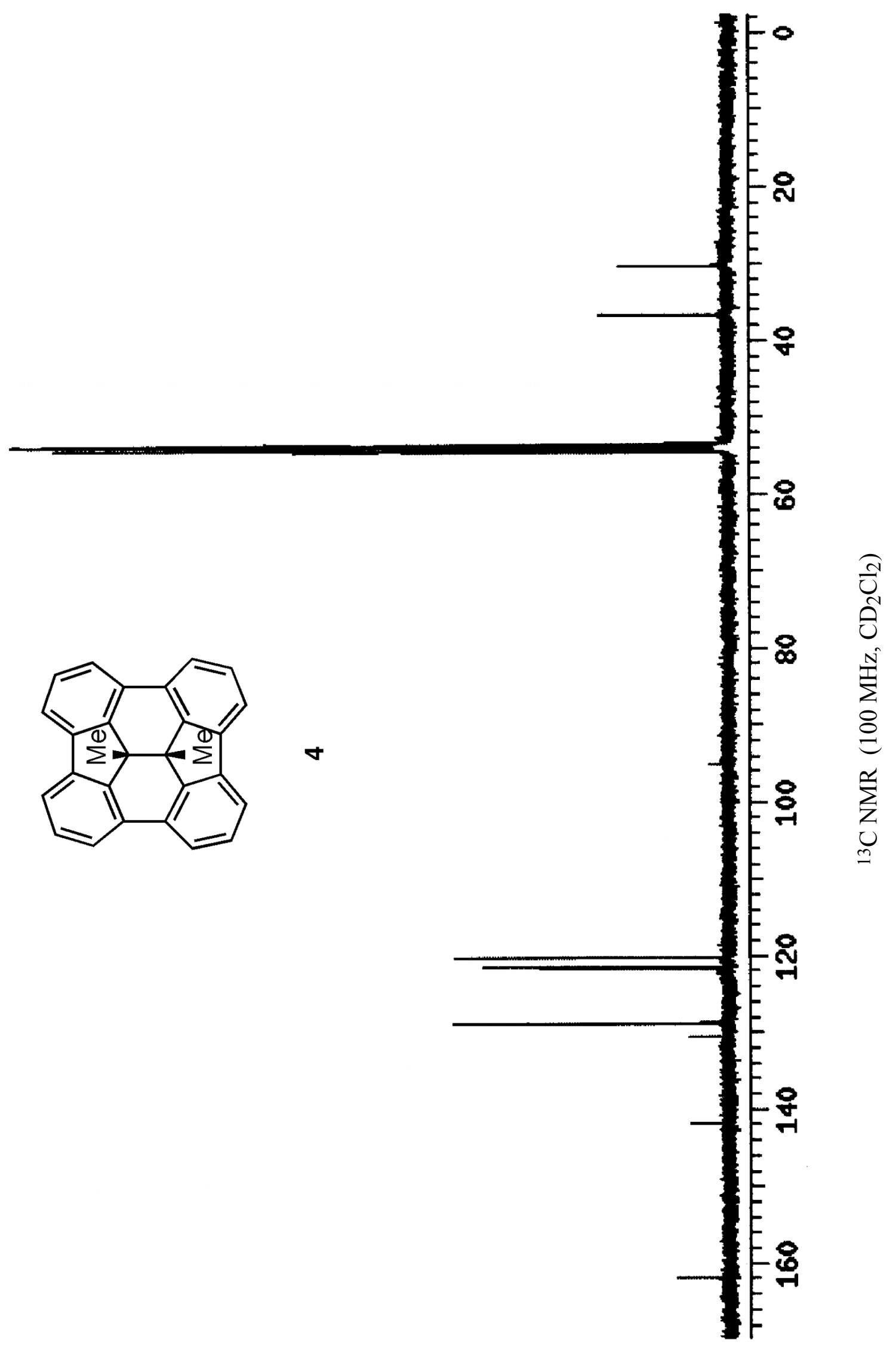




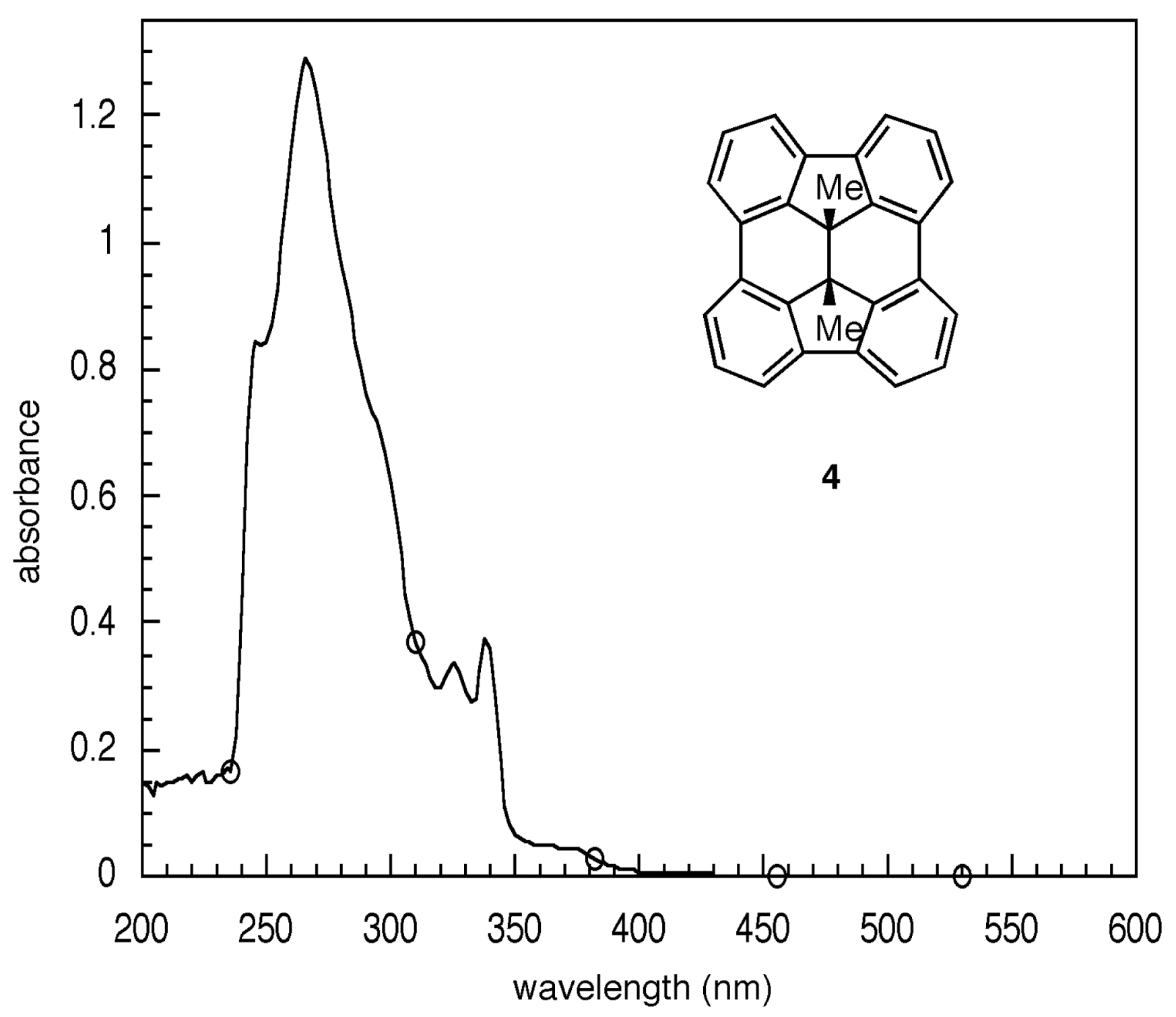

UV-VIS $\left(\mathrm{CHCl}_{3}\right)$ 

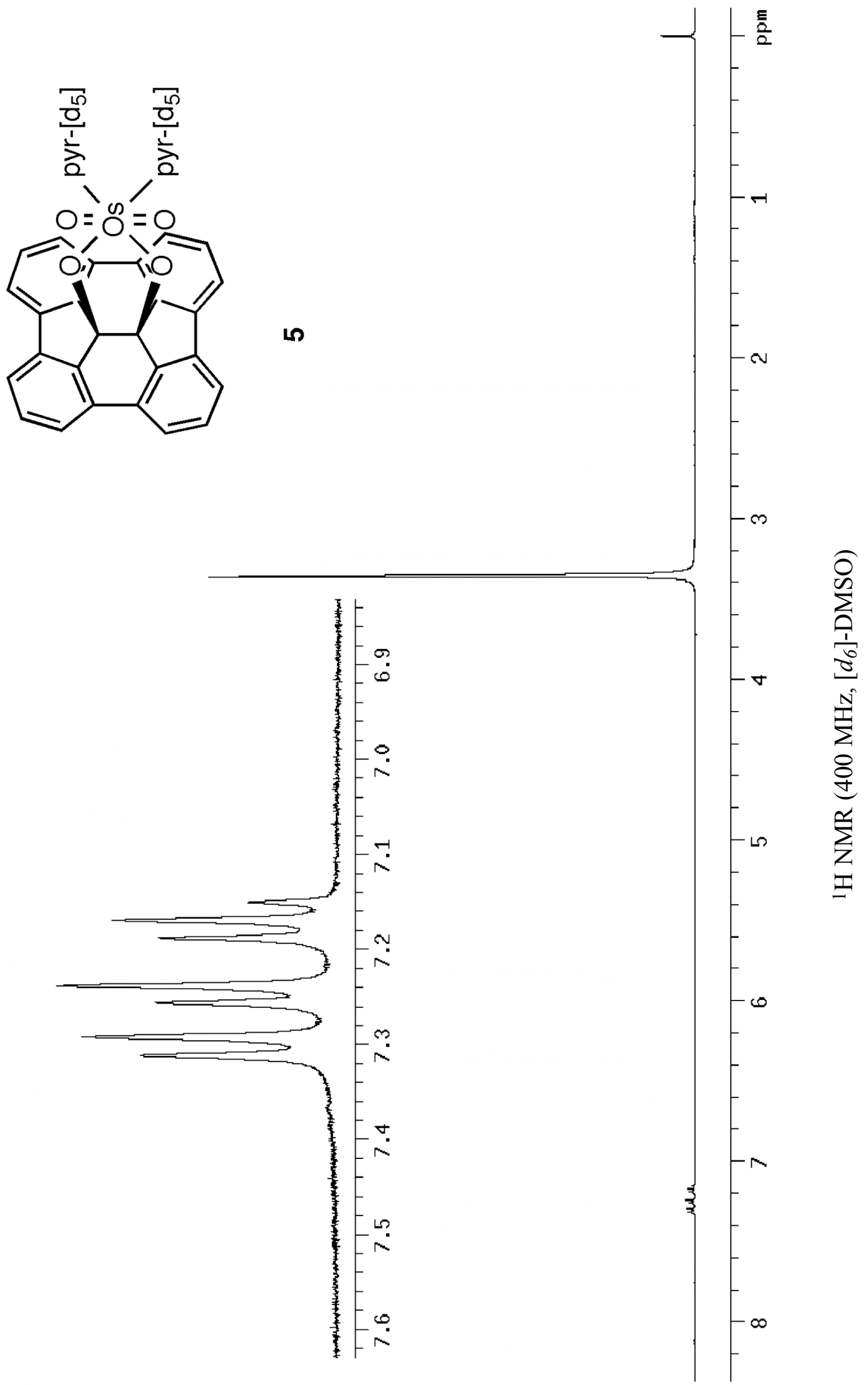
Calculated DFT (B3LYP/6-31G*) energy and X,Y,Z-coordinates for compound 1

\begin{tabular}{|c|c|c|c|c|}
\hline \multicolumn{5}{|c|}{ Coordinates (Angstroms) } \\
\hline \multicolumn{2}{|c|}{ АтОМ } & $\mathrm{x}$ & $\mathrm{Y}$ & z \\
\hline 1 & $\mathrm{H}$ & 4.535268 & 1.595596 & 0.519058 \\
\hline 2 & $\mathrm{C}$ & 3.518649 & 1.775896 & 0.178877 \\
\hline 3 & $\mathrm{C}$ & 2.703875 & 0.761804 & -0.308245 \\
\hline 4 & $\mathrm{H}$ & 3.618052 & 3.871085 & 0.669968 \\
\hline 5 & $\mathrm{C}$ & 1.393895 & -1.164352 & -0.695927 \\
\hline 6 & $\mathrm{C}$ & 1.393895 & 1.164352 & -0.695927 \\
\hline 7 & $\mathrm{C}$ & 0.749235 & 2.394736 & -0.441985 \\
\hline 8 & $\mathrm{C}$ & 0.668292 & 0.000000 & -0.998916 \\
\hline 9 & $\mathrm{C}$ & -1.633964 & -3.393582 & 0.033495 \\
\hline 10 & $\mathrm{C}$ & 1.633964 & 3.393582 & 0.033495 \\
\hline 11 & $\mathrm{C}$ & 2.972825 & 3.081765 & 0.292759 \\
\hline 12 & $\mathrm{H}$ & 1.278461 & 4.397378 & 0.251459 \\
\hline 13 & $\mathrm{C}$ & -0.749235 & 2.394736 & -0.441985 \\
\hline 14 & $\mathrm{C}$ & -2.703875 & 0.761804 & -0.308245 \\
\hline 15 & $\mathrm{C}$ & 3.518649 & -1.775896 & 0.178877 \\
\hline 16 & $\mathrm{H}$ & 4.535268 & -1.595596 & 0.519058 \\
\hline 17 & $\mathrm{C}$ & 2.703875 & -0.761804 & -0.308245 \\
\hline 18 & $\mathrm{H}$ & 3.618052 & -3.871085 & 0.669968 \\
\hline 19 & $\mathrm{C}$ & 0.749235 & -2.394736 & -0.441985 \\
\hline 20 & $\mathrm{C}$ & -0.668292 & 0.000000 & -0.998916 \\
\hline 21 & $\mathrm{H}$ & -1.278461 & -4.397378 & 0.251459 \\
\hline 22 & $\mathrm{C}$ & 1.633964 & -3.393582 & 0.033495 \\
\hline 23 & $\mathrm{C}$ & 2.972825 & -3.081765 & 0.292759 \\
\hline 24 & $\mathrm{H}$ & 1.278461 & -4.397378 & 0.251459 \\
\hline 25 & $\mathrm{C}$ & -1.393895 & -1.164352 & -0.695927 \\
\hline 26 & $\mathrm{C}$ & -1.393895 & 1.164352 & -0.695927 \\
\hline 27 & $\mathrm{C}$ & -0.749235 & -2.394736 & -0.441985 \\
\hline 28 & $\mathrm{C}$ & -2.703875 & -0.761804 & -0.308245 \\
\hline 29 & $\mathrm{C}$ & -3.518649 & 1.775896 & 0.178877 \\
\hline 30 & $\mathrm{C}$ & -3.518649 & -1.775896 & 0.178877 \\
\hline 31 & $\mathrm{C}$ & -2.972825 & 3.081765 & 0.292759 \\
\hline 32 & $\mathrm{H}$ & -4.535268 & 1.595596 & 0.519058 \\
\hline 33 & $\mathrm{C}$ & -1.633964 & 3.393582 & 0.033495 \\
\hline 34 & $\mathrm{H}$ & -3.618052 & 3.871085 & 0.669968 \\
\hline 35 & $\mathrm{H}$ & -3.618052 & -3.871085 & 0.669968 \\
\hline 36 & $\mathrm{H}$ & -1.278461 & 4.397378 & 0.251459 \\
\hline 37 & $\mathrm{C}$ & -2.972825 & -3.081765 & 0.292759 \\
\hline 38 & $\mathrm{H}$ & -4.535268 & -1.595596 & 0.519058 \\
\hline Poin & nt Group: & $\mathrm{c} 2 \mathrm{~V} \quad$ Number & of degrees of & E freedom: \\
\hline
\end{tabular}


Calculated DFT (B3LYP/6-31G*) energy and X,Y,Z-coordinates for compound 4

\begin{tabular}{|c|c|c|c|c|}
\hline \multicolumn{5}{|c|}{ Coordinates (Angstroms) } \\
\hline \multicolumn{2}{|c|}{ АТОМ } & $x$ & $\mathrm{Y}$ & $\mathrm{z}$ \\
\hline 1 & $\mathrm{H}$ & 4.343877 & 1.460949 & 1.325365 \\
\hline 2 & $\mathrm{C}$ & 3.385403 & 1.709855 & 0.877557 \\
\hline 3 & $\mathrm{C}$ & 2.603904 & 0.750364 & 0.234568 \\
\hline 4 & $\mathrm{H}$ & 3.467071 & 3.771040 & 1.498359 \\
\hline 5 & $\mathrm{C}$ & 1.376417 & -1.157072 & -0.326378 \\
\hline 6 & $\mathrm{C}$ & 1.376417 & 1.157072 & -0.326378 \\
\hline 7 & $\mathrm{C}$ & 0.752399 & 2.370601 & -0.032968 \\
\hline 8 & $\mathrm{C}$ & 0.767542 & 0.000000 & -1.073336 \\
\hline 9 & $\mathrm{C}$ & -1.563712 & -3.328791 & 0.613155 \\
\hline 10 & $\mathrm{C}$ & 1.563712 & 3.328791 & 0.613155 \\
\hline 11 & $\mathrm{C}$ & 2.868743 & 3.011633 & 1.001871 \\
\hline 12 & $\mathrm{H}$ & 1.160222 & 4.302888 & 0.875746 \\
\hline 13 & $\mathrm{C}$ & -0.752399 & 2.370601 & -0.032968 \\
\hline 14 & $\mathrm{C}$ & -2.603904 & 0.750364 & 0.234568 \\
\hline 15 & $\mathrm{C}$ & 3.385403 & -1.709855 & 0.877557 \\
\hline 16 & $\mathrm{H}$ & 4.343877 & -1.460949 & 1.325365 \\
\hline 17 & $\mathrm{C}$ & 2.603904 & -0.750364 & 0.234568 \\
\hline 18 & $\mathrm{H}$ & 3.467071 & -3.771040 & 1.498359 \\
\hline 19 & $\mathrm{C}$ & 0.752399 & -2.370601 & -0.032968 \\
\hline 20 & $\mathrm{C}$ & -0.767542 & 0.000000 & -1.073336 \\
\hline 21 & $\mathrm{H}$ & -1.160222 & -4.302888 & 0.875746 \\
\hline 22 & $\mathrm{C}$ & 1.563712 & -3.328791 & 0.613155 \\
\hline 23 & $\mathrm{C}$ & 2.868743 & -3.011633 & 1.001871 \\
\hline 24 & $\mathrm{H}$ & 1.160222 & -4.302888 & 0.875746 \\
\hline 25 & $\mathrm{C}$ & -1.376417 & -1.157072 & -0.326378 \\
\hline 26 & $\mathrm{C}$ & -1.376417 & 1.157072 & -0.326378 \\
\hline 27 & $\mathrm{C}$ & -0.752399 & -2.370601 & -0.032968 \\
\hline 28 & C & -2.603904 & -0.750364 & 0.234568 \\
\hline 29 & $\mathrm{C}$ & -3.385403 & 1.709855 & 0.877557 \\
\hline 30 & $\mathrm{C}$ & -3.385403 & -1.709855 & 0.877557 \\
\hline 31 & $\mathrm{C}$ & -2.868743 & 3.011633 & 1.001871 \\
\hline 32 & $\mathrm{H}$ & -4.343877 & 1.460949 & 1.325365 \\
\hline 33 & $\mathrm{C}$ & -1.563712 & 3.328791 & 0.613155 \\
\hline 34 & $\mathrm{H}$ & -3.467071 & 3.771040 & 1.498359 \\
\hline 35 & $\mathrm{H}$ & -3.467071 & -3.771040 & 1.498359 \\
\hline 36 & $\mathrm{H}$ & -1.160222 & 4.302888 & 0.875746 \\
\hline 37 & $\mathrm{C}$ & -2.868743 & -3.011633 & 1.001871 \\
\hline 38 & $\mathrm{H}$ & -4.343877 & -1.460949 & 1.325365 \\
\hline 39 & $\mathrm{C}$ & -1.403965 & 0.000000 & -2.503919 \\
\hline 40 & $\mathrm{C}$ & 1.403965 & 0.000000 & -2.503919 \\
\hline 41 & $\mathrm{H}$ & 1.112221 & -0.889402 & -3.069845 \\
\hline 42 & $\mathrm{H}$ & 2.493341 & 0.000000 & -2.417602 \\
\hline 43 & $\mathrm{H}$ & 1.112221 & 0.889402 & -3.069845 \\
\hline 44 & $\mathrm{H}$ & -2.493341 & 0.000000 & -2.417602 \\
\hline 45 & $\mathrm{H}$ & -1.112221 & -0.889402 & -3.069845 \\
\hline 46 & $\mathrm{H}$ & -1.112221 & 0.889402 & -3.069845 \\
\hline
\end{tabular}

Point Group: $\mathrm{c} 2 \mathrm{v}$ Number of degrees of freedom:

Energy is $\quad-1077.799640865$ 
Calculated DFT (B3LYP/6-31G*) energy and X,Y,Z-coordinates for compound 11

\begin{tabular}{|c|c|c|c|c|}
\hline \multicolumn{5}{|c|}{ Coordinates (Angstroms) } \\
\hline \multicolumn{2}{|c|}{ АTOM } & $\mathrm{x}$ & $\mathrm{Y}$ & $\mathrm{z}$ \\
\hline 1 & $\mathrm{H}$ & 4.580411 & 0.728248 & -1.592533 \\
\hline 2 & $\mathrm{C}$ & 3.560450 & 0.390465 & -1.777849 \\
\hline 3 & $\mathrm{C}$ & 2.735776 & -0.044579 & -0.738484 \\
\hline 4 & $\mathrm{H}$ & 3.700873 & 0.802506 & -3.890030 \\
\hline 5 & $\mathrm{C}$ & 1.417923 & -0.498685 & 1.130539 \\
\hline 6 & $\mathrm{C}$ & 1.417923 & -0.498685 & -1.130539 \\
\hline 7 & $\mathrm{C}$ & 0.818605 & -0.273923 & -2.402327 \\
\hline 8 & $\mathrm{C}$ & 0.715102 & -0.876125 & 0.000000 \\
\hline 9 & $\mathrm{C}$ & -1.481836 & 0.489587 & 3.315406 \\
\hline 10 & $\mathrm{C}$ & 1.695137 & 0.185250 & -3.394656 \\
\hline 11 & $\mathrm{C}$ & 3.048234 & 0.454192 & -3.091197 \\
\hline 12 & $\mathrm{H}$ & 1.337332 & 0.393804 & -4.402853 \\
\hline 13 & $\mathrm{C}$ & -0.677108 & -0.193592 & -2.372017 \\
\hline 14 & $\mathrm{C}$ & -2.553922 & 0.078061 & -0.752542 \\
\hline 15 & $\mathrm{C}$ & 3.560450 & 0.390465 & 1.777849 \\
\hline 16 & $\mathrm{H}$ & 4.580411 & 0.728248 & 1.592533 \\
\hline 17 & $\mathrm{C}$ & 2.735776 & -0.044579 & 0.738484 \\
\hline 18 & $\mathrm{H}$ & 3.700873 & 0.802506 & 3.890030 \\
\hline 19 & $\mathrm{C}$ & 0.818605 & -0.273923 & 2.402327 \\
\hline 20 & $\mathrm{C}$ & -0.704971 & -1.220603 & 0.000000 \\
\hline 21 & $\mathrm{H}$ & -1.061475 & 0.775939 & 4.277356 \\
\hline 22 & $\mathrm{C}$ & 1.695137 & 0.185250 & 3.394656 \\
\hline 23 & $\mathrm{C}$ & 3.048234 & 0.454192 & 3.091197 \\
\hline 24 & $\mathrm{H}$ & 1.337332 & 0.393804 & 4.402853 \\
\hline 25 & $\mathrm{C}$ & -1.341318 & -0.514161 & 1.166039 \\
\hline 26 & $\mathrm{C}$ & -1.341318 & -0.514161 & -1.166039 \\
\hline 27 & $\mathrm{C}$ & -0.677108 & -0.193592 & 2.372017 \\
\hline 28 & C & -2.553922 & 0.078061 & 0.752542 \\
\hline 29 & $\mathrm{C}$ & -3.313788 & 0.763454 & -1.701952 \\
\hline 30 & $\mathrm{C}$ & -3.313788 & 0.763454 & 1.701952 \\
\hline 31 & $\mathrm{C}$ & -2.779194 & 0.903586 & -2.997667 \\
\hline 32 & $\mathrm{H}$ & -4.260969 & 1.235717 & -1.449268 \\
\hline 33 & $\mathrm{C}$ & -1.481836 & 0.489587 & -3.315406 \\
\hline 34 & $\mathrm{H}$ & -3.356713 & 1.438302 & -3.750132 \\
\hline 35 & $\mathrm{H}$ & -3.356713 & 1.438302 & 3.750132 \\
\hline 36 & $\mathrm{H}$ & -1.061475 & 0.775939 & -4.277356 \\
\hline 37 & $\mathrm{C}$ & -2.779194 & 0.903586 & 2.997667 \\
\hline 38 & $\mathrm{H}$ & -4.260969 & 1.235717 & 1.449268 \\
\hline 39 & C & -0.990736 & -2.748949 & 0.000000 \\
\hline 40 & $\mathrm{H}$ & -2.069280 & -2.958173 & 0.000000 \\
\hline 41 & $\mathrm{H}$ & -0.543475 & -3.212245 & 0.887592 \\
\hline 42 & $\mathrm{H}$ & -0.543475 & -3.212245 & -0.887592 \\
\hline Poi & & : cs Number & of degrees & f freedom: \\
\hline
\end{tabular}

Energy is $\quad-1037.936368592$ 
Calculated DFT (B3LYP/6-31G*) energy and X,Y,Z-coordinates for compound 12

\begin{tabular}{|c|c|c|c|c|}
\hline \multicolumn{5}{|c|}{ Coordinate } \\
\hline \multicolumn{2}{|c|}{ АTOM } & $\mathrm{x}$ & $\mathrm{Y}$ & z \\
\hline 1 & $\mathrm{H}$ & 4.633116 & 0.195902 & -1.575277 \\
\hline 2 & $\mathrm{C}$ & 3.565268 & 0.241368 & -1.773674 \\
\hline 3 & C & 3.091965 & -0.005304 & -3.074354 \\
\hline 4 & $\mathrm{H}$ & 1.441515 & -0.410877 & -4.403841 \\
\hline 5 & $\mathrm{C}$ & 1.723660 & -0.101319 & 3.400446 \\
\hline 6 & $\mathrm{C}$ & 1.723660 & -0.101319 & -3.400446 \\
\hline 7 & C & 0.752548 & 0.132506 & -2.413025 \\
\hline 8 & C & 0.401019 & 0.651021 & 0.000000 \\
\hline 9 & $\mathrm{C}$ & -1.723660 & 0.101319 & 3.400446 \\
\hline 10 & $\mathrm{C}$ & 1.283638 & 0.517362 & -1.169245 \\
\hline 11 & C & 2.626046 & 0.485346 & -0.759567 \\
\hline 12 & $\mathrm{H}$ & 3.819310 & -0.198087 & 3.859023 \\
\hline 13 & $\mathrm{C}$ & -0.752548 & -0.132506 & -2.413025 \\
\hline 14 & C & -2.626046 & -0.485346 & -0.759567 \\
\hline 15 & C & 3.565268 & 0.241368 & 1.773674 \\
\hline 16 & $\mathrm{H}$ & 4.633116 & 0.195902 & 1.575277 \\
\hline 17 & $\mathrm{C}$ & 3.091965 & -0.005304 & 3.074354 \\
\hline 18 & $\mathrm{H}$ & 1.441515 & -0.410877 & 4.403841 \\
\hline 19 & C & 0.752548 & 0.132506 & 2.413025 \\
\hline 20 & C & -0.401019 & -0.651021 & 0.000000 \\
\hline 21 & $\mathrm{H}$ & -1.441515 & 0.410877 & 4.403841 \\
\hline 22 & $\mathrm{C}$ & 1.283638 & 0.517362 & 1.169245 \\
\hline 23 & C & 2.626046 & 0.485346 & 0.759567 \\
\hline 24 & C & -0.392781 & 1.981602 & 0.000000 \\
\hline 25 & $\mathrm{C}$ & -1.283638 & -0.517362 & 1.169245 \\
\hline 26 & $\mathrm{C}$ & -1.283638 & -0.517362 & -1.169245 \\
\hline 27 & $\mathrm{C}$ & -0.752548 & -0.132506 & 2.413025 \\
\hline 28 & C & -2.626046 & -0.485346 & 0.759567 \\
\hline 29 & C & -3.565268 & -0.241368 & -1.773674 \\
\hline 30 & C & -3.565268 & -0.241368 & 1.773674 \\
\hline 31 & C & -3.091965 & 0.005304 & -3.074354 \\
\hline 32 & $\mathrm{H}$ & -4.633116 & -0.195902 & -1.575277 \\
\hline 33 & $\mathrm{C}$ & -1.723660 & 0.101319 & -3.400446 \\
\hline 34 & $\mathrm{H}$ & -3.819310 & 0.198087 & -3.859023 \\
\hline 35 & $\mathrm{H}$ & -3.819310 & 0.198087 & 3.859023 \\
\hline 36 & $\mathrm{H}$ & -1.441515 & 0.410877 & -4.403841 \\
\hline 37 & C & -3.091965 & 0.005304 & 3.074354 \\
\hline 38 & $\mathrm{H}$ & -4.633116 & -0.195902 & 1.575277 \\
\hline 39 & C & 0.392781 & -1.981602 & 0.000000 \\
\hline 40 & $\mathrm{H}$ & -0.327902 & -2.806295 & 0.000000 \\
\hline 41 & $\mathrm{H}$ & 1.021922 & -2.084687 & 0.887205 \\
\hline 42 & $\mathrm{H}$ & 1.021922 & -2.084687 & -0.887205 \\
\hline 43 & $\mathrm{H}$ & 3.819310 & -0.198087 & -3.859023 \\
\hline 44 & $\mathrm{H}$ & -1.021922 & 2.084687 & -0.887205 \\
\hline 45 & $\mathrm{H}$ & 0.327902 & 2.806295 & 0.000000 \\
\hline 46 & $\mathrm{H}$ & -1.021922 & 2.084687 & 0.887205 \\
\hline
\end{tabular}

Point Group: $\mathrm{c} 2 \mathrm{~h}$ Number of degrees of freedom:

Energy is $\quad-1077.802661434$ 
Calculated DFT (B3LYP/6-31G*) energy and X,Y,Z-coordinates for compound 13

\begin{tabular}{|c|c|c|c|c|}
\hline \multicolumn{5}{|c|}{ Coordinates (Angstroms) } \\
\hline \multicolumn{2}{|c|}{ АTOM } & $\mathrm{x}$ & $\mathrm{Y}$ & $\mathrm{z}$ \\
\hline 1 & C & 0.696542 & 0.000000 & 0.000000 \\
\hline 2 & $\mathrm{C}$ & -0.696542 & 0.000000 & 0.000000 \\
\hline 3 & $\mathrm{C}$ & -1.430229 & 1.259703 & 0.000000 \\
\hline 4 & C & -1.430229 & -1.259703 & 0.000000 \\
\hline 5 & $\mathrm{C}$ & 1.430229 & -1.259703 & 0.000000 \\
\hline 6 & $\mathrm{C}$ & 1.430229 & 1.259703 & 0.000000 \\
\hline 7 & $\mathrm{C}$ & -2.849382 & -3.697115 & 0.000000 \\
\hline 8 & C & -0.729442 & -2.498779 & 0.000000 \\
\hline 9 & $\mathrm{C}$ & -2.846632 & -1.275703 & 0.000000 \\
\hline 10 & $\mathrm{C}$ & -3.535900 & -2.480771 & 0.000000 \\
\hline 11 & C & -1.467287 & -3.700593 & 0.000000 \\
\hline 12 & $\mathrm{H}$ & -4.623961 & 2.470346 & 0.000000 \\
\hline 13 & $\mathrm{H}$ & -3.396841 & 4.635674 & 0.000000 \\
\hline 14 & $\mathrm{H}$ & -4.623961 & -2.470346 & 0.000000 \\
\hline 15 & $\mathrm{H}$ & -0.955029 & -4.654771 & 0.000000 \\
\hline 16 & $\mathrm{H}$ & -3.396841 & -4.635674 & 0.000000 \\
\hline 17 & $\mathrm{C}$ & 2.849382 & -3.697115 & 0.000000 \\
\hline 18 & $\mathrm{C}$ & 2.846632 & -1.275703 & 0.000000 \\
\hline 19 & C & 0.729442 & -2.498779 & 0.000000 \\
\hline 20 & C & 1.467287 & -3.700593 & 0.000000 \\
\hline 21 & $\mathrm{C}$ & 3.535900 & -2.480771 & 0.000000 \\
\hline 22 & $\mathrm{H}$ & -4.318962 & 0.000000 & -0.871863 \\
\hline 23 & $\mathrm{H}$ & 4.318962 & 0.000000 & -0.871863 \\
\hline 24 & $\mathrm{H}$ & 0.955029 & -4.654771 & 0.000000 \\
\hline 25 & $\mathrm{H}$ & 4.623961 & -2.470346 & 0.000000 \\
\hline 26 & $\mathrm{H}$ & 3.396841 & -4.635674 & 0.000000 \\
\hline 27 & $\mathrm{C}$ & 2.849382 & 3.697115 & 0.000000 \\
\hline 28 & $\mathrm{C}$ & 0.729442 & 2.498779 & 0.000000 \\
\hline 29 & $\mathrm{C}$ & 2.846632 & 1.275703 & 0.000000 \\
\hline 30 & $\mathrm{C}$ & 3.535900 & 2.480771 & 0.000000 \\
\hline 31 & $\mathrm{C}$ & 1.467287 & 3.700593 & 0.000000 \\
\hline 32 & $\mathrm{H}$ & 4.318962 & 0.000000 & 0.871863 \\
\hline 33 & $\mathrm{C}$ & 3.647665 & 0.000000 & 0.000000 \\
\hline 34 & $\mathrm{H}$ & 4.623961 & 2.470346 & 0.000000 \\
\hline 35 & $\mathrm{H}$ & 0.955029 & 4.654771 & 0.000000 \\
\hline 36 & $\mathrm{H}$ & 3.396841 & 4.635674 & 0.000000 \\
\hline 37 & $\mathrm{C}$ & -2.849382 & 3.697115 & 0.000000 \\
\hline 38 & $\mathrm{C}$ & -2.846632 & 1.275703 & 0.000000 \\
\hline 39 & $\mathrm{C}$ & -0.729442 & 2.498779 & 0.000000 \\
\hline 40 & $\mathrm{C}$ & -1.467287 & 3.700593 & 0.000000 \\
\hline 41 & $\mathrm{C}$ & -3.535900 & 2.480771 & 0.000000 \\
\hline 42 & $\mathrm{C}$ & -3.647665 & 0.000000 & 0.000000 \\
\hline 43 & $\mathrm{H}$ & -4.318962 & 0.000000 & 0.871863 \\
\hline 44 & $\mathrm{H}$ & -0.955029 & 4.654771 & 0.000000 \\
\hline Poin & ht Group & : $\mathrm{d} 2 \mathrm{~h} \quad$ Number & of degrees of & E freedom: \\
\hline
\end{tabular}


Calculated DFT (B3LYP/6-31G*) energy and X,Y,Z-coordinates for compound 14

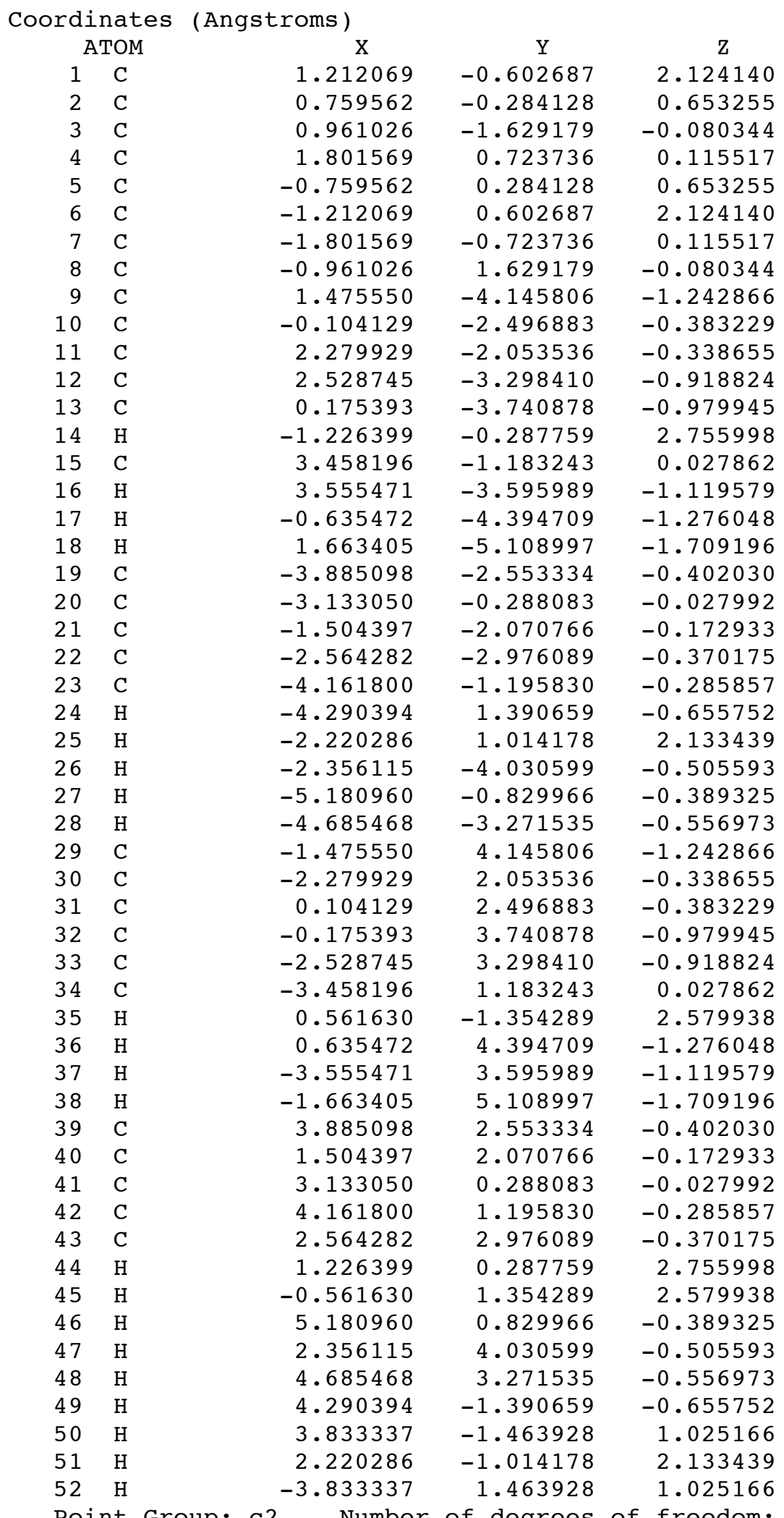

Point Group: c2 Number of degrees of freedom: 
Calculated DFT (B3LYP/6-31G*) energy and X,Y,Z-coordinates for $\mathrm{C}_{60}$

\begin{tabular}{|c|c|c|c|c|c|c|c|c|c|}
\hline \multicolumn{10}{|c|}{ Coordinates (Angstroms) } \\
\hline \multicolumn{2}{|c|}{ АTOM } & $\mathrm{X}$ & $\mathrm{Y}$ & $\mathrm{Z}$ & & & & & \\
\hline 1 & $\mathrm{C}$ & 3.464241 & -0.776407 & -0.028142 & 31 & $\mathrm{C}$ & -3.017875 & 1.449190 & -1.180446 \\
\hline 2 & C & 3.482309 & 0.652961 & 0.234114 & 32 & $\mathrm{C}$ & -1.777327 & 3.064946 & 0.211564 \\
\hline 3 & $\mathrm{C}$ & 3.101639 & 1.550337 & -0.764504 & 33 & $\mathrm{C}$ & -2.759893 & 0.435540 & -2.189754 \\
\hline 4 & $\mathrm{C}$ & 2.759893 & -0.435540 & 2.189754 & 34 & $\mathrm{C}$ & -2.687015 & -1.055454 & 2.066640 \\
\hline 5 & C & 1.599082 & 1.894513 & -2.540721 & 35 & $\mathrm{C}$ & -3.464241 & 0.776407 & 0.028142 \\
\hline 6 & $\mathrm{C}$ & 2.269687 & 2.695345 & -0.433609 & 36 & $\mathrm{C}$ & -0.042085 & -2.647826 & -2.363500 \\
\hline 7 & $\mathrm{C}$ & 1.851819 & 2.897553 & 0.882543 & 37 & $\mathrm{C}$ & -1.277568 & 2.206315 & 2.469717 \\
\hline 8 & $\mathrm{C}$ & 1.340995 & 2.907846 & -1.531461 & 38 & $\mathrm{C}$ & -3.066582 & 1.251544 & 1.278479 \\
\hline 9 & $\mathrm{C}$ & -1.686490 & 0.583608 & -3.069289 & 39 & $\mathrm{C}$ & -3.482309 & -0.652961 & -0.234114 \\
\hline 10 & $\mathrm{C}$ & 2.248437 & 1.963067 & 1.922588 & 40 & $\mathrm{C}$ & -2.669840 & 0.316964 & 2.318464 \\
\hline 11 & $\mathrm{C}$ & 3.046975 & 0.863567 & 1.604879 & 41 & $\mathrm{C}$ & -1.129940 & -1.808909 & -2.838003 \\
\hline 12 & $\mathrm{C}$ & 1.129940 & 1.808909 & 2.838003 & 42 & $\mathrm{C}$ & -3.046975 & -0.863567 & -1.604879 \\
\hline 13 & $\mathrm{C}$ & 3.066582 & -1.251544 & -1.278479 & 43 & $\mathrm{C}$ & 0.825946 & -1.751335 & 2.975263 \\
\hline 14 & C & 2.191758 & -2.570070 & 1.090350 & 44 & $\mathrm{C}$ & 3.017875 & -1.449190 & 1.180446 \\
\hline 15 & $\mathrm{C}$ & 2.669840 & -0.316964 & -2.318464 & 45 & $\mathrm{C}$ & -1.564189 & 0.907111 & 3.054511 \\
\hline 16 & $\mathrm{C}$ & 2.205995 & -2.419208 & -1.372042 & 46 & $\mathrm{C}$ & 0.854555 & 0.561384 & 3.399865 \\
\hline 17 & $\mathrm{C}$ & 2.687015 & 1.055454 & -2.066640 & 47 & $\mathrm{C}$ & 1.686490 & -0.583608 & 3.069289 \\
\hline 18 & $\mathrm{C}$ & 1.277568 & -2.206315 & -2.469717 & 48 & $\mathrm{C}$ & -0.519781 & 0.101474 & 3.510007 \\
\hline 19 & $\mathrm{C}$ & 0.537519 & 1.327896 & -3.247734 & 49 & $\mathrm{C}$ & -0.488293 & -3.320848 & -1.155167 \\
\hline 20 & C & 0.031854 & 3.314129 & -1.269606 & 50 & $\mathrm{C}$ & -1.599082 & -1.894513 & 2.540721 \\
\hline 21 & $\mathrm{C}$ & 0.042085 & 2.647826 & 2.363500 & 51 & $\mathrm{C}$ & -0.537519 & -1.327896 & 3.247734 \\
\hline 22 & $\mathrm{C}$ & 0.519781 & -0.101474 & -3.510007 & 52 & $\mathrm{C}$ & -1.340995 & -2.907846 & 1.531461 \\
\hline 23 & $\mathrm{C}$ & 1.564189 & -0.907111 & -3.054511 & 53 & $\mathrm{C}$ & -1.851819 & -2.897553 & -0.882543 \\
\hline 24 & $\mathrm{C}$ & -0.854555 & -0.561384 & -3.399865 & 54 & $\mathrm{C}$ & 1.073625 & -2.724366 & 2.006089 \\
\hline 25 & $\mathrm{C}$ & -1.073625 & 2.724366 & -2.006089 & 55 & $\mathrm{C}$ & -3.101639 & -1.550337 & 0.764504 \\
\hline 26 & C & -0.403172 & 3.524947 & 0.101008 & 56 & $\mathrm{C}$ & -2.248437 & -1.963067 & -1.922588 \\
\hline 27 & $\mathrm{C}$ & -0.825946 & 1.751335 & -2.975263 & 57 & $\mathrm{C}$ & -2.269687 & -2.695345 & 0.433609 \\
\hline 28 & $\mathrm{C}$ & -2.191758 & 2.570070 & -1.090350 & 58 & $\mathrm{C}$ & 0.403172 & -3.524947 & -0.101008 \\
\hline 29 & $\mathrm{C}$ & 0.488293 & 3.320848 & 1.155167 & 59 & $\mathrm{C}$ & -0.031854 & -3.314129 & 1.269606 \\
\hline 30 & C & -2.205995 & 2.419208 & 1.372042 & 60 & $\mathrm{C}$ & 1.777327 & -3.064946 & -0.211564 \\
\hline
\end{tabular}

Point Group: ci Number of degrees of freedom: 87

Energy is $\quad-2286.173691677$ 
Calculated DFT (B3LYP/6-31G*) energy and X,Y,Z-coordinates for 1,2-dimethyl-C 60

\begin{tabular}{|c|c|c|c|c|c|c|c|c|c|}
\hline \multicolumn{10}{|c|}{ Coordinates (Angstroms) } \\
\hline & ТОМ & $\mathrm{x}$ & $\mathrm{Y}$ & $\mathrm{Z}$ & & & & & \\
\hline 1 & $\mathrm{C}$ & 2.313851 & 2.595407 & -0.065177 & 35 & $\mathrm{C}$ & -2.311027 & -2.600077 & 1.386014 \\
\hline 2 & $\mathrm{C}$ & 2.311027 & 2.600077 & 1.386014 & 36 & $\mathrm{C}$ & -1.429132 & 1.174596 & -2.378023 \\
\hline 3 & $\mathrm{C}$ & 1.177535 & 3.028032 & 2.081009 & 37 & $\mathrm{C}$ & 0.727995 & -2.304336 & 3.254267 \\
\hline 4 & $\mathrm{C}$ & 3.485915 & 0.696961 & 0.665035 & 38 & $\mathrm{C}$ & -1.177535 & -3.028032 & 2.081009 \\
\hline 5 & $\mathrm{C}$ & -1.177535 & 3.028032 & 2.081009 & 39 & $\mathrm{C}$ & -2.313851 & -2.595407 & -0.065177 \\
\hline 6 & $\mathrm{C}$ & 0.727995 & 2.304336 & 3.254267 & 40 & $\mathrm{C}$ & 0.000000 & -3.469299 & 1.350150 \\
\hline 7 & $\mathrm{C}$ & 1.425911 & 1.175633 & 3.690121 & 41 & $\mathrm{C}$ & -2.572705 & 0.725478 & -1.631180 \\
\hline 8 & $\mathrm{C}$ & -0.727995 & 2.304336 & 3.254267 & 42 & $\mathrm{C}$ & -3.030934 & -1.425396 & -0.508644 \\
\hline 9 & $\mathrm{C}$ & -3.485915 & 0.696961 & 0.665035 & 43 & $\mathrm{C}$ & 3.030934 & -1.425396 & -0.508644 \\
\hline 10 & $\mathrm{C}$ & 2.603309 & 0.727027 & 2.968053 & 44 & $\mathrm{C}$ & 3.030934 & 1.425396 & -0.508644 \\
\hline 11 & $\mathrm{C}$ & 3.038282 & 1.425085 & 1.839644 & 45 & $\mathrm{C}$ & 1.177535 & -3.028032 & 2.081009 \\
\hline 12 & $\mathrm{C}$ & 2.603309 & -0.727027 & 2.968053 & 46 & $\mathrm{C}$ & 3.038282 & -1.425085 & 1.839644 \\
\hline 13 & $\mathrm{C}$ & 1.176367 & 3.005010 & -0.769935 & 47 & $\mathrm{C}$ & 3.485915 & -0.696961 & 0.665035 \\
\hline 14 & $\mathrm{C}$ & 2.572705 & 0.725478 & -1.631180 & 48 & $\mathrm{C}$ & 2.311027 & -2.600077 & 1.386014 \\
\hline 15 & $\mathrm{C}$ & 0.000000 & 3.450184 & -0.044814 & 49 & $\mathrm{C}$ & -0.811178 & 0.000000 & -3.166317 \\
\hline 16 & $\mathrm{C}$ & 0.740363 & 2.284560 & -1.946230 & 50 & $\mathrm{C}$ & 1.176367 & -3.005010 & -0.769935 \\
\hline 17 & $\mathrm{C}$ & 0.000000 & 3.469299 & 1.350150 & 51 & $\mathrm{C}$ & 2.313851 & -2.595407 & -0.065177 \\
\hline 18 & $\mathrm{C}$ & -0.740363 & 2.284560 & -1.946230 & 52 & $\mathrm{C}$ & 0.740363 & -2.284560 & -1.946230 \\
\hline 19 & $\mathrm{C}$ & -2.311027 & 2.600077 & 1.386014 & 53 & $\mathrm{C}$ & -1.429132 & -1.174596 & -2.378023 \\
\hline 20 & $\mathrm{C}$ & -1.425911 & 1.175633 & 3.690121 & 54 & $\mathrm{C}$ & 2.572705 & -0.725478 & -1.631180 \\
\hline 21 & $\mathrm{C}$ & 1.425911 & -1.175633 & 3.690121 & 55 & $\mathrm{C}$ & -1.176367 & -3.005010 & -0.769935 \\
\hline 22 & $\mathrm{C}$ & -2.313851 & 2.595407 & -0.065177 & 56 & $\mathrm{C}$ & -2.572705 & -0.725478 & -1.631180 \\
\hline 23 & $\mathrm{C}$ & -1.176367 & 3.005010 & -0.769935 & 57 & $\mathrm{C}$ & -0.740363 & -2.284560 & -1.946230 \\
\hline 24 & $\mathrm{C}$ & -3.030934 & 1.425396 & -0.508644 & 58 & $\mathrm{C}$ & 0.811178 & 0.000000 & -3.166317 \\
\hline 25 & $\mathrm{C}$ & -2.603309 & 0.727027 & 2.968053 & 59 & $\mathrm{C}$ & 1.429132 & -1.174596 & -2.378023 \\
\hline 26 & $\mathrm{C}$ & -0.697987 & 0.000000 & 4.137041 & 60 & $\mathrm{C}$ & 1.429132 & 1.174596 & -2.378023 \\
\hline 27 & $\mathrm{C}$ & -3.038282 & 1.425085 & 1.839644 & 61 & $\mathrm{C}$ & 1.416057 & 0.000000 & -4.598071 \\
\hline 28 & $\mathrm{C}$ & -2.603309 & -0.727027 & 2.968053 & 62 & $\mathrm{C}$ & -1.416057 & 0.000000 & -4.598071 \\
\hline 29 & $\mathrm{C}$ & 0.697987 & 0.000000 & 4.137041 & 63 & $\mathrm{H}$ & 1.120238 & -0.889553 & -5.160967 \\
\hline 30 & $\mathrm{C}$ & -0.727995 & -2.304336 & 3.254267 & 64 & $\mathrm{H}$ & 2.507855 & 0.000000 & -4.525962 \\
\hline 31 & $\mathrm{C}$ & -3.038282 & -1.425085 & 1.839644 & 65 & $\mathrm{H}$ & 1.120238 & 0.889553 & -5.160967 \\
\hline 32 & $\mathrm{C}$ & -1.425911 & -1.175633 & 3.690121 & 66 & $\mathrm{H}$ & -2.507855 & 0.000000 & -4.525962 \\
\hline 33 & $\mathrm{C}$ & -3.485915 & -0.696961 & 0.665035 & 67 & $\mathrm{H}$ & -1.120238 & -0.889553 & -5.160967 \\
\hline 34 & $\mathrm{C}$ & 0.000000 & -3.450184 & -0.044814 & 68 & $\mathrm{H}$ & -1.120238 & 0.889553 & -5.160967 \\
\hline
\end{tabular}

Point Group: c2v Number of degrees of freedom: 53

Energy is $\quad-2366.000631007$ 